
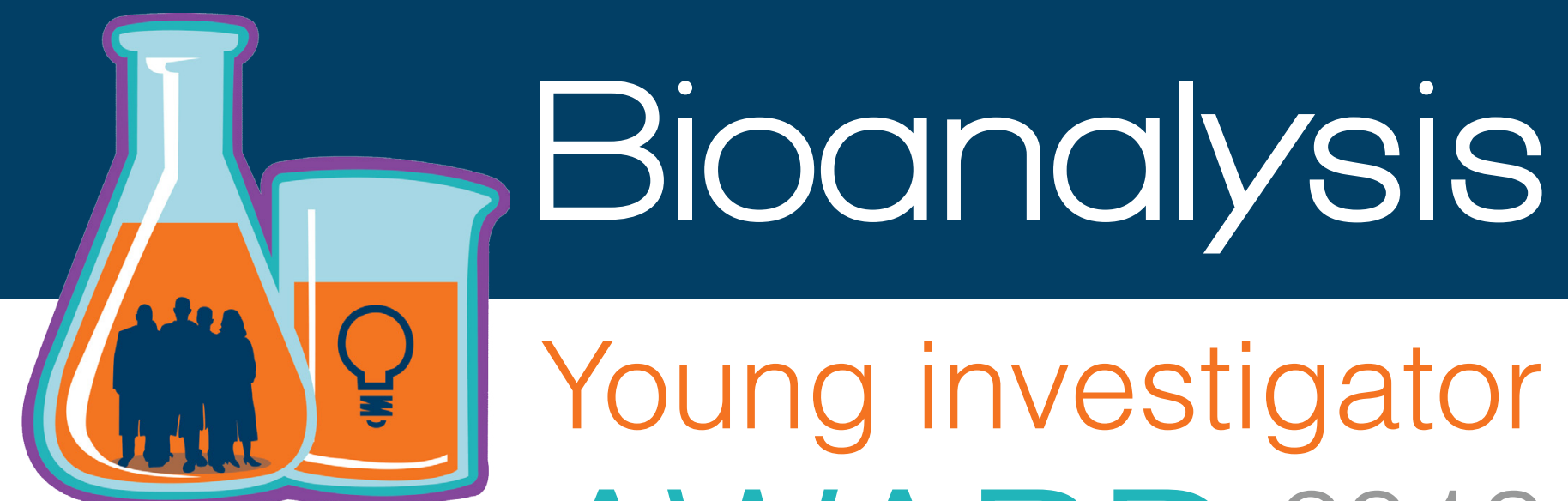

Young investigator

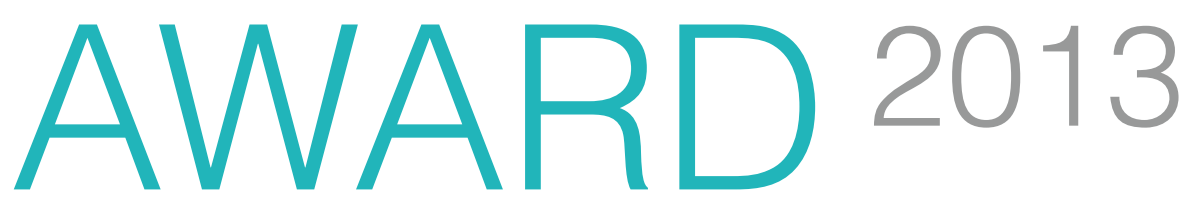

Each year, Bioanalysis and Bioanalysis Zone run the Young Investigator Award to identify and reward promising early-career researchers in our community.

This year has seen the largest number of nominees yet, with 18 young scientists in the running to win the 2013 Award!

Held in association with Waters and the European Bioanalysis Forum

\section{Waters}

THE SCIENCE OF WHAT'S POSSIBLE.'

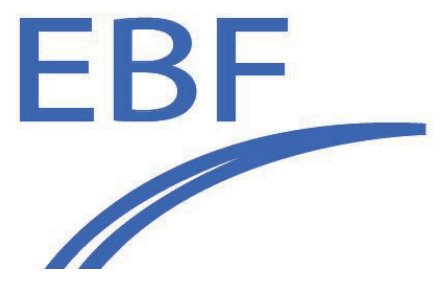

We are pleased to announce that this year's Award will again be held in association with Waters and the European Bioanalysis Forum, with the winner receiving US $\$ 1000$, a year's subscription to Bioanalysis and free open access for their next article published in the journal. They will also receive help with travel costs to ensure they can accept their award in person at the European Bioanalysis Forum Symposium in November, where they will have the chance to make a short presentation on their work.

We will be publishing full profiles of all 18 nominees on Bioanalysis Zone (www.bioanalysis-zone.com) and summary profiles will published across four issues of Bioanalysis.

Once all the profiles have been published, our Editorial board will help us to narrow the field to five finalists before we open our online vote and ask you to choose our winner.

\section{Make sure you don't miss out on the latest news and your chance to vote!}

\title{
FUTURE SCIENCE
}




\section{Supporting comments}

I am recommending Yong Zeng based on his research achievements as a graduate student and postdoctorate, and his first year as a faculty member at the University of Kansas

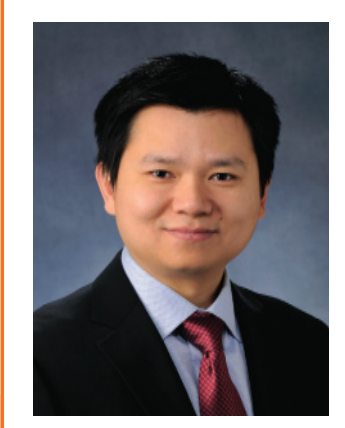
Yong Zeng (KU). Yong's research is multidisciplinary and takes place at the intersection of analytical chemistry and nanotechnology with biology and medicine. During his PhD studies, he pioneered the exploration of self-assembled nanomaterials for microfluidic bioseparations and proteomics. As a postdoctoral associate, Yong developed novel methodology for sequencing DNA from single cells, which was highlighted in Science and many other journals. At KU, Yong continues to work on innovative bioanalytical microtechnologies to address challenging biomedical problems including the development of novel devices to study the role of glycomics in profiling of disease. Most recently, he has been working with investigators at the KU cancer center to develop microfluidic-based methods for early detection of cancer. In summary, I feel Yong is an outstanding example of a young rising star in the bioanalysis field and is most deserving of the distinction of the Bioanalysis Young Investigator Award 2013.

Nominated by: Susan Lunte, University of Kansas, Multidisciplinary Research Building, 2030 Becker Drive, Room 210, Lawrence, KS 66047-I620, USA Tel.: +I 78586438 II; Fax: +I 785864 1916; E-mail: slunte@ku.edu

\section{Financial \& competing interests disclosure}

$Y$ Zeng has no relevant affiliations or financial involvement with any organization or entity with a financial interest in or financial conflict with the subject matter or materials discussed in the manuscript. This includes employment, consultancies, honoraria, stock ownership or options, expert testimony, grants or patents received or pending, or royalties.

No writing assistance was utilized in the production of this manuscript.
Q Describe the main highlights of your bioanalytical research, \& its importance to the bioanalytical community, both now \& in the future.

My $\mathrm{PhD}$ research was focused on developing microfluidic approaches to control colloidal self-assembly to fabricate ordered porous nanostructures. This work led to the first demonstration of self-assembled nanosieves for efficient and fast bioseparations. In contrast to conventional nanolithography, the self-assembly method features ease of fabrication, reduced cost and the ability of large-scale patterning of 3D nanostructures. Precise control of local fluid-dynamics enabled by the microfluidic strategy affords a conceptually new route to create a whole new class of nanostructures inaccessible for conventional bulk scale self-assembly means. These unique nanostructures provide well-defined confinement to elucidate dynamics and transport of biomolecules, opening new opportunities for developing novel separation mechanisms.

During my postdoctoral research, I developed a microfluidic single-cell genetic analysis technology and demonstrated its feasibility for preclinical detection of leukemia. Early-stage cancer is challenging to study partially because most current methods lack the ability to examine low-frequency mutant cells and thus require ensemble measurement of a large cell population which can mask important information of cellular individuality. Single-cell genetic analysis provides a high-throughput platform for quantitative detection and single molecule sequencing of mutations at extremely low frequency. This technology can be adapted to other single-cell biochemical measurements of cell behaviors such as cellular response to drugs.

\section{Where do you see your career in} bioanalysis taking you?

One of my current research focuses is to develop high-throughput microfluidics for massively parallel, quantitative single cell analysis of tumor cells by seamlessly integrating single-cell capture and sensitive molecular assays. This research aims to yield transformative tools to facilitate cancer biology, pre-symptomatic diagnosis, and monitoring of disease relapse. I am also exploiting microfluidic principles to develop next-generation glycomic technologies. Despite the potential of protein glycosylation for cancer diagnosis and prognosis, progress in glycomics has largely lagged behind proteomics due to the complexity of glycome and dynamic glycan alteration in patients. Our approach represents a paradigm shift from conventional benchtop methods, to develop an automated microfluidic lectin array platform. This new tool will substantially aid in obtaining integrative and dynamic overview of cancer development and developing novel glyco-biomarkers. I envision my long-term career path involving active collaborations with investigators from University of Kansas Medical Center and other institutes. 


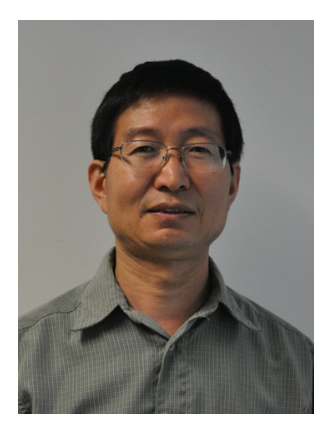

\section{Wanlong Zhou}

US FDA, 5I00 Paint Branch Parkway HFS-7I7 College Park,
MD 20740-3835, USA
Tel.: +I 240 402 I862
Fax: +I 707 37I 3779
E-mail: wanlong.zhou@fda.hhs.gov

US FDA, 5I00 Paint Branch Parkway HFS-7I7 College Park,
MD 20740-3835, USA
Tel.: +I 240 402 I862
Fax: +I 707 37I 3779
E-mail: wanlong.zhou@fda.hhs.gov

US FDA, 5I00 Paint Branch Parkway HFS-7I7 College Park,
MD 20740-3835, USA
Tel.: +I 240 402 I862
Fax: +I 707 37I 3779
E-mail: wanlong.zhou@fda.hhs.gov

US FDA, 5I00 Paint Branch Parkway HFS-7I7 College Park,
MD 20740-3835, USA
Tel.: +I 240 402 I862
Fax: +I 707 37I 3779
E-mail: wanlong.zhou@fda.hhs.gov

US FDA, 5I00 Paint Branch Parkway HFS-7I7 College Park,
MD 20740-3835, USA
Tel.: +I 240 402 I862
Fax: +I 707 37I 3779
E-mail: wanlong.zhou@fda.hhs.gov

.

\section{Supporting comments}

Dr Zhou brings skills in problem solving and instrumentation with regard to analytical chemistry to the cosmetic group that the other staff members do not have. He also serves as a 'go-to' person on instrumentation when other staff members within the group are having problems. One of Dr Zhou's major projects was developing and publishing a sensitive method for quantitating the hexapeptides AC-EEMQRR-amide and $\mathrm{H}_{2} \mathrm{~N}$-EEMQRR-amide in cosmetics advertised for their anti-wrinkle properties. This method made it possible to perform animal studies aimed at determining whether, and how far, such peptides penetrate into the skin. The major impact on our US FDA mission related to this project is whether the incorporation of these specific peptides should help define these products as drugs or cosmetics, or combination drug-cosmetic products. This research impacts on public health with regard to the safety of these products to consumers.

Nominated by: Alexander J Krynitsky, Chief, Bioanalytical Methods Branch, US FDA, Center for Food Safety \& Applied Nutrition, Office of Regulatory Science, Division of Bioanalytical Chemistry, 5100 Paint Branch, HFS-717 College Park, MD 20740-3835, USA

Tel.: + I 240402 2098; Fax: + 301436 2332; E-mail: alex.krynitsky@fda.hhs.gov

Q Describe the main highlights of your bioanalytical research, \& its importance to the bioanalytical community, both now \& in the future.

Development of rapid analytical methods for in vitro skin penetration studies of peptides is challenging because of the need for high sensitivity and accurate quantification of peptides in complex skin samples and vehicle matrices. To date, only a few HPLC-UV methods have been reported for the analysis of peptides for skin penetration studies. I developed and fully validated the first hydrophilic interaction LC-MS/MS (HILIC) method for analysis of the peptides Ac-EEMQRR-amide and $\mathrm{H}_{2} \mathrm{~N}$-EEMQRR-amide. This method was then used for the determination of these peptides in various complex matrices. A HILIC-SPE procedure was developed to minimize significant ion suppression that would affect quantification in the more complex sample matrices. In addition, stable isotopically labeled peptides, corresponding to the above native peptides, were used to compensate for these matrix effects. To the best of our knowledge, this was the first method that used HILIC-MS/MS, HILIC-SPE in combination with stable isotopically labeled standards to overcome matrix effects and provide accurate quantification in these complex sample matrices. Both the first phase and the second phase of the study were published in J. Chromatogr. A (Vol: 1218, Issue: 44) and Bioanalysis (Vol: 5 Issue: 11), respectively. The third phase is in preparation for publication.
How do you envisage the field of bioanalysis evolving in the future?

In addition to increased complexity of sample matrices from in vitro and in vivo studies, these studies are usually costly and samples are limited. LC-HRMS, such as TOF and Orbitrap MS, especially Q-TOF and Q-Orbitrap MS, will play a more important role in the future. For the quantitative and qualitative analysis, these instruments have the ability to analyze a virtually unlimited number of 'compounds' because they operate in full-scan mode and can reconstruct any desired ion chromatograms using the same full-scan data file without re-analyzing the samples. For analyzing possible metabolites and other unknown compounds, an online library search of high-resolution mass spectra may be performed because accurate mass measurements are almost specific and universal for each analyte of interest, regardless of the instrumentation used. High-resolution MS (QTOF or Q-Orbitrap) provides not only accurate-mass measurements and high full-scan sensitivity but also additional features such as structure elucidation and confirmation. The accurate product ion spectra can be obtained by performing MS/ MS experiments using (QTOF or Q-Orbitrap) MS and used to search compound libraries to confirm the structures of compounds. This is crucial for identifying metabolites or unknown compounds when the reference standards are not available.

\section{Financial \& competing interests disclosure \\ WZhou has no relevant affliations or financial involvement with any organization or entity with a financial interest in or financial conflict with the subject matter or materials discussed in the manuscript. This includes employment, consultancies, honoraria, stock ownership or options, expert testimony, grants or patents received or pending, or royalties. \\ No writing assistance was utilized in the production of this manuscript.}




\section{Supporting comments}

I am very pleased to nominate Justo Giner Martínez-Sierra for the Bioanalysis Young Investigator Award 2013. Justo has very recently finished his $\mathrm{PhD}$ thesis under my supervision (January

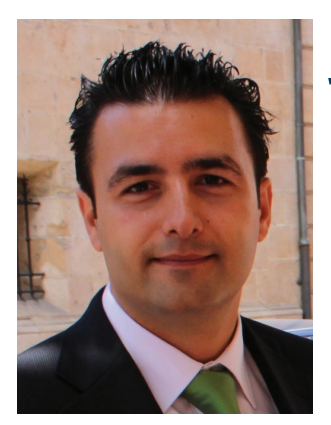

Justo Giner Martínez-Sierra

2013). His outstanding thesis 'Development of analytical methodologies for the study of sulfur metabolism using enriched stable isotopes' is an example of multidisciplinary work (analytical chemistry, biochemistry and biotechnology of yeast, animal experiments, spectroscopy, chemometrics, etc.) that involves the use of state-of-the-art bioanalytical technology. His work has been very challenging, but also exciting and rewarding for. The overall quality of the work is excellent. During his PhD Justo was able to prepare eight scientific manuscripts (all SCI ranked). In five papers he is the first author; all were accepted in excellent analytical journals with impact factors over 3. Some of the published research includes results obtained in collaboration with international organisations including LGC Limited and the UK National Measurement Institute for Chemical and Biological Measurements, where Justo carried out two pre-doctoral stays. His list of conference contributions is impressive, with eight poster presentations and ten oral presentations (three as presenting author), all of which were at international conferences of high prestige. I am confident that Justo will achieve even greater feats in times to come and strongly recommend his candidature for the Bioanalysis Young Investigator Award 2013.

Nominated by: José Ignacio García Alonso, University of Oviedo, Department of Physical \& Analytical Chemistry, c/ Julián Clavería, 8, 33006, Oviedo, Spain Tel.: +34 985103 484; Fax: +34 985103 125; E-mail: jiga@uniovi.es

\section{Financial \& competing interests disclosure}

JG Martinez-Sierra has no relevant affliations or financial involvement with any organization or entity with a financial interest in or financial confict with the subject matter or materials discussed in the manuscript. This includes employment, consultancies, honoraria, stock ownership or options, expert testimony, grants or patents received or pending, or royalties.

No writing assistance was utilized in the production of this manuscript.
Q Describe the main highlights of your bioanalytical research, \& its importance to the bioanalytical community, both now \& in the future.

My research is focused on novel analytical Inductively Coupled Plasma-MS (ICP-MS)based concepts with great potential in bioanalysis. We have labeled proteins in yeast with enriched sulphur-34 and investigated different ICP-MS platforms in combination with chromatographic separation methods for $S$-tracer studies. The introduced tool set using multiple tracers (sulphur-33 for quantification purposes) enabled us to actually measure the isotopic ratio and the concentration of the most important S-species (e.g. amino acids methionine and cysteine) in the yeast, and therefore could be used for quantitative proteomics (S-containing peptides and proteins) using isotope dilution analysis. This successful fundamental work was the essential starting point to highlight completely new ways for studying S-metabolism using stable and nonradioactive tracers, opening the way to in vivo studies of S-metabolism. In the same vein, we have developed methodologies by HPLC-ICP-MS to carry out iron and selenium metabolism studies using enriched stable isotopes.

Additionally, in connection with natural variations of the isotopic composition, we have developed a method for the measurement of longitudinal variations of $\mathrm{S}$-isotope ratios in single human-hair strands by Laser AblationICP-MS that could potentially aid prediction of geographical origin and recent movements of subjects, or provide information on diet and lifestyle.

Describe the most difficult challenge you have encountered in the laboratory and how you overcame it?

I have faced so many challenges in my professional life, but it is not easy to categorize them into difficult, more difficult or most difficult. Challenges are challenges! In my opinion, the important thing is how I deal with it and I always try to overcome it with energy, positive attitude and self confidence.

Currently, I am on the way to becoming a biotech entrepreneur. That means a lot of interdisciplinary work from outside my scientific background, that's why I am trying to improve the ability to 'speak' both business and science, taking the time to understand the marketing, financial, business issues and needs of the other disciplines that are relevant to my goals. Anyway, I have learnt that everyday is a challenge, in fact, life itself is a challenge. I enjoy the challenges, so I enjoy the life. 


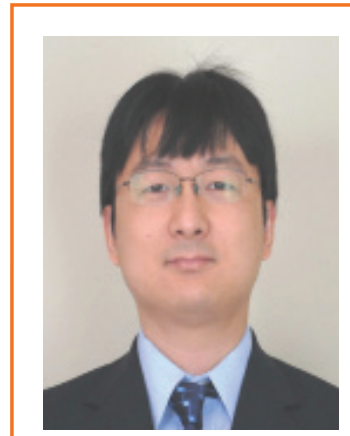

\section{Long Yuan}

Bioanalytical Sciences, Research \& Development, BristolMyers Squibb, Route 206 \& Province Line Road, Princeton,

NJ 08543, USA

Tel.: + I 6092525336

E-mail: long.yuan@bms.com

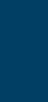

\section{Supporting comments}

I am pleased to recommend Long Yuan for the Bioanalysis Young Investigator Award 2013. After obtaining his PhD in Richard van Breemen's laboratory, he joined the regulated bioanalysis group at Bristol-Myers Squibb. He quickly adapted to the industry research environment and has made tremendous contributions in method development, validation and sample analysis to support multiple promising drug-development programs. He is one of the main contributors in providing bioanalytical support in developing drugs to treat and potentially cure HCV infections. The assays he developed for pre-clinical study support involves multiple therapeutic compounds and different biological matrices, including various types of tissues. In addition to regular project support, Long also enthusiastically explores new technology and new methodology for bioanalysis. Particularly, he has been actively working on implementing automation in his daily work, as well as in new bioanalytical frontiers. His automation of standard and QC preparation for DBS assays was highly innovative and epitomizes his approach to streamlining bioanalytical work and his attention to safety. He further investigated an improved sample preparation strategy for LC-MS/MS assay for proteins, which has been adopted for multiple assays in project support. Long is very willing to help others and share his knowledge with colleagues and the broader bioanalytical scientific community. This is well-reflected in his recent multiple publications in Bioanalysis and other peer-reviewed journals. Long certainly has established himself as a role model for young scientists who pursue their career in this exciting scientific field.

Nominated by: Qin Ji, Bioanalytical Sciences, Research \& Development, Bristol-Myers Squibb, Route 206 and Province Line Road, Princeton, NJ 08543, USA Tel.: + 609252 5560; E-mail: qin.ji@bms.com

Q Describe the main highlights of your bioanalytical research, \& its importance to the bioanalytical community, both now \& in the future.

For LC-MS/MS-based bioanalysis of proteins, achieving an efficient and reproducible digestion to generate the surrogate peptide(s) is critical. I investigated the pellet digestion methodology, which is a simple and fast digestion process without time-consuming pretreatment steps. I demonstrated that pellet digestion provided similar or better digestion efficiency for the test protein, even for the hard-to-digest regions, compared with traditional digestion methods. I also investigated the SPE clean-up strategy to improve the protein assay sensitivity, and found it was an efficient and easy-to-develop method to remove background peptides. I evaluated and successfully applied the use of a robotic liquid handler to automatically prepare standard and QC samples in blood and to spot the blood samples onto DBS cards. This automated process can significantly improve efficiency, robustness and safety of DBS bioanalysis, and is easy to apply in other laboratories. In another work, I systematically evaluated the root cause of non-linearity in LC-MS/MS bioanalytical assays and developed a strategy to predict and extend the linear standard curve range.
Q Describe the most difficult challenge you have encountered in the laboratory and how you overcame it?

The most difficult challenge for me has been the quantitative analysis of a monoclonal antibody using LC-MS/MS; especially since I had no experience in protein analysis. I dived into the literature, consulted with experienced colleagues, and more importantly, spent a lot of time conducting experiments. I identified some areas that I could work on to improve the efficiency and applicability of LC-MS/MS bioanalysis of proteins. As previously mentioned, I evaluated pellet digestion methodology and successfully applied this in the quantitative analysis of a monoclonal antibody in serum. Another key challenge for LC-MS/ MS-based assays is sensitivity. The highly abundant endogenous proteins in serum are digested together with the target protein and large amounts of background peptides with similar chemical properties to the surrogate peptides are generated. Sample clean-up is critical for achieving a highly sensitive assay. I worked on strategies to improve sensitivity. I evaluated SPE clean-up methods and demonstrated that the orthogonal SCX SPE/RP UHPLC approach can efficiently remove most background peptides and improve assay sensitivity (manuscript submitted to Bioanalysis).
Financial \& competing interests disclosure

$L$ Yuan has no relevant affliations or financial involvement with any organization or entity with a financial interest in or financial conflict with the subject matter or materials discussed in the manuscript. This includes employment, consultancies, honoraria, stock ownership or options, expert testimony, grants or patents received or pending, or royalties.

No writing assistance was utilized in the production of this manuscript. 